\title{
A case of tension-type headache in fibromyalgia
}

\author{
Giuseppina Stifano - Stefania Colantuono - Valentina Carusi \\ Fabiola La Marra · Alessandro Marra • Massimo Granata
}

Received: 23 February 2010/Accepted: 19 April 2010/Published online: 9 May 2010

(C) Springer-Verlag 2010

Sir,

A 57-year-old-woman was admitted to our ward for a daily tension-type headache, it was not responding to usual pharmacological treatment. Before 5 years, she had hysterectomy and after 10 days, she had a domestic accident reporting an injury of the pelvis and the spine. Since then, she begun to suffer of muscular rigor of the neck and the shoulder girdle, daily, intense and constrictive pain localized in the occipital spine, not associated with vomiting, ocular symptoms, not worsened by exercise. The diagnosis of daily tension-type headache met the IHCD-II criteria. She also reported weakness of the upper and lower limbs, tingling, tremors and difficulties in walking and climbing. The patient's medical history reported: Raynaud phenomenon, chronic gastritis and dysphagia, hepatitis $\mathrm{C}$ virus infection, hyperhomocystenemia, homozygosis for MTHFR 677C factor. She also was a heavy smoker. She referred widespread pain, unusually severe above all joints and muscular pain, without any sign of inflammation at clinical examination. The patient underwent a brain and neck CT, a brain angio-MRI, a color-Doppler examination of the epiaortic and transcranial vessels and blood tests that resulted negative. Ultrasonography of soft tissues showed fibrosis of the shoulder muscles with involvement of the dermis and hypodermis. Since then, the headache could be a symptom of a systemic autoimmune disease, we decided to perform

G. Stifano $\cdot$ S. Colantuono $\cdot$ V. Carusi .

F. La Marra - A. Marra - M. Granata $(\bowtie)$

Headache Centre, U.O.C. Clinical Immunology,

Sapienza Università di Roma, Rome, Italy

e-mail: massimo.granata@uniroma1.it several immunological tests that showed positive result for ANA and negative to ENA, anti-ds DNA, AMA, ASMA, APCA, ANCA. We also performed an electromyography and a muscle and skin biopsy of the deltoid muscle which resulted negative. In addition, the patient was examined by a neurologist and a psychiatrist and she underwent a personality test, which emphasized a state of mild depression. The differential diagnosis excluded systemic diseases of connective tissue (systemic sclerosis, mixed connectivity, polymyositis and systemic lupus erythematosus), therefore, the most probable diagnosis is tension-type headache in HCV-related fibromyalgia [1-4] (Fig. 1). The diagnosis of fibromyalgia was made according to the ACR criteria and calculating widespread pain index and symptom severity (SS) scale of by Wolfe et al. [7]. The patient had pain caused by digital palpation of the neck, shoulder, chest, hip, knee and elbow regions. The patient has been treated with antidepressants, anxiolytics and antiepileptic GABAergic drugs with an improvement of the symptoms.

Fibromyalgia is a multifactorial disease of unknown etiology. At the moment, it is considered as a neurosensitive disorder characterized by abnormal processing of pain by the SNC. In our case, the daily tension-type headache certainly was a symptom of fibromyalgic syndrome. There are many common features between the two pathologies: the symptomatology often overlaps and the origin of pain is regarded as "central"; indeed, the same neurotransmitters (serotonin, glutamate, $\mathrm{P}$ substance, CGRP) and receptors are involved [5, 6]. If the pathogenetic hypothesis is the same, also the pharmacological treatment is common. Antidepressants (SSRI), antiepileptics and anxiolytics are used in both cases, and likewise are useful muscle relaxation techniques. In our opinion, fibromyalgia should be always considered, especially in women between 20 and 40 years, in differential diagnosis of headache. In our case, 


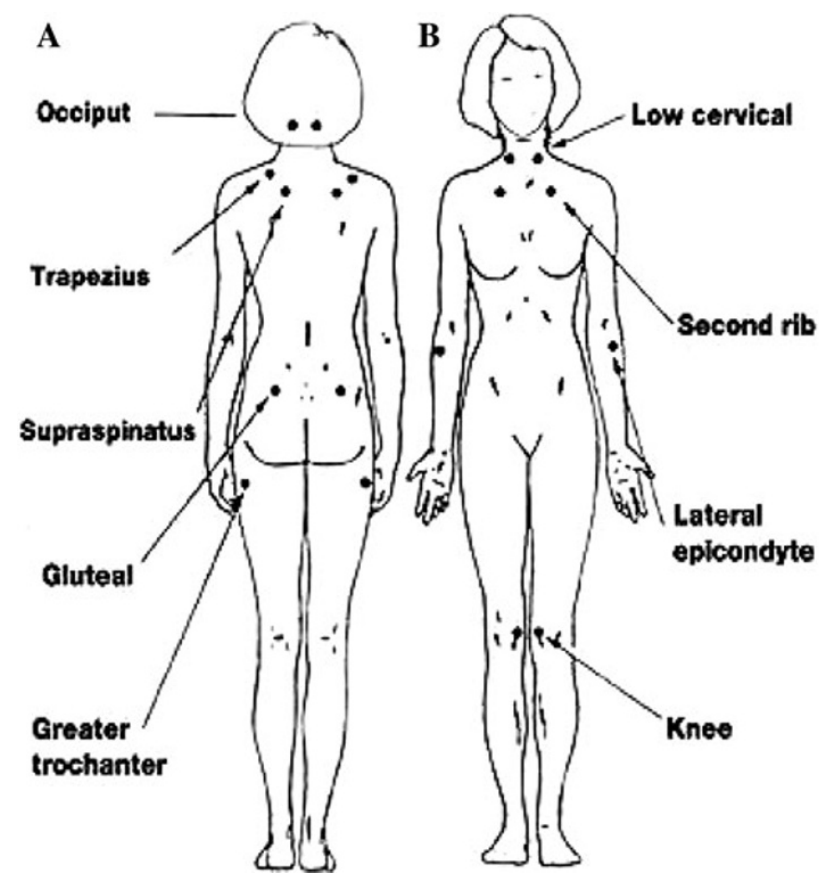

Fig. 1 ACR fibromyalgia 1990. a Widespread pain, b pain caused by digital palpation of tender points in at least 11 of 18 locations

headache and fibromyalgia seem to appear in the same period, there is a correlation or it is a causality?
Conflict of interest None.

\section{References}

1. De Tommaso M, Sardaro M, Serpino C, Costantini F, Vecchio E, Prudenzano MP, Lamberti P, Livrea P (2009) Fibromyalgia comorbidity in primary headaches. Cephalalgia 29(4):453-464

2. Palazzi C, D'Amico E, D'Angelo S, Nucera A, Petricca A, Olivieri I (2008) Hepatitis $C$ virus infection in Italian patients with fibromyalgia. Clin Rheumatol 27:101-103

3. Schoenen J (2004) Tension-type headache and fibromyalgia: what's common, what's different? Neurol Sci 25:S157-S159

4. Dawn A. Marcus, Cheryl Bernstein, Rudy TE (2005) Fibromyalgia and headache: an epidemiological study supporting migraine as part of the fibromyalgia syndrome. Clin Rheumatol 24:595-601

5. Leistad RB, Nilsen KB, Stovner LJ, Westgaard RH, Rø M, Sand T (2008) Similarities in stress physiology among patients with chronic pain and headache disorders: evidence for a common pathophysiological mechanism? J Headache Pain 9(3):165-175

6. Bradley LA (2009) Pathophysiology of fibromyalgia. Am J Med 122:S22-S30

7. Wolfe $F$ et al (2010) New ACR criteria for fibromyalgia-a twenty-years journey. Arthritis Care Res 62(5):600-610 